\title{
Electric field of a 2D elliptical charge distribution inside a cylindrical conductor
}

\author{
Miguel A. Furman* \\ Center for Beam Physics, Lawrence Berkeley National Laboratory, Building 71R0259, 1 Cyclotron Road, \\ Berkeley, California 94720-8211, USA \\ (Received 18 May 2007; published 7 August 2007)
}

\begin{abstract}
By combining the method of images with calculus of complex variables, we provide a simple expression for the electric field of a two-dimensional (2D) static elliptical charge distribution inside a perfectly conducting circular cylinder. The charge distribution need not be concentric with the cylinder.

PACS numbers: $41.20 . \mathrm{Cv}$
\end{abstract}

\section{INTRODUCTION}

Many applications in beam physics, particularly those concerning transverse beam dynamics, call for an approximate computation of the transverse electric field produced by the charge distribution of the beam, namely, the field components in the plane perpendicular to the beam motion. Mathematically, this approximation becomes exact in the limit of an infinitely long charge distribution that is also constant in the longitudinal direction. In practice, the approximation is a reasonable starting point in many cases. Examples include proton beams used in spallation neutron sources, or heavy-ion fusion ion beams, in which the characteristic length of variation of the charge density along the longitudinal direction is much larger than the transverse beam size. In this case the longitudinal component of the electric field is much smaller than the transverse, hence the field is effectively contained in the $2 \mathrm{D}$ transverse plane. Another example arises in the case of ultrarelativistic beams, for which the electric field is effectively squeezed into a 2D transverse "pancake" owing to the Lorentz contraction of the longitudinal component of the field. Furthermore, the approximation of an elliptical charge distribution, as defined in Sec. II below, is also a reasonable starting point for numerous beam dynamics problems both for lepton and hadron beams. A few recent examples can be found in Refs. [1-3].

In Ref. [4] we developed a formalism to compute the 2D electric field for elliptical charge distributions in free space. The formalism makes essential use of Cauchy's theorem, and yields a simple and quite general formula for the field in complex form. In this article we extend the formalism to elliptical distributions contained inside a perfectly conducting circular cylinder by applying the method of images.

Our formalism naturally yields the electric field itself $\mathbf{E}$ rather than the electric potential $\Phi$. The fundamental reason is that, in two dimensions, $\mathbf{E}$ has a dependence on distance $r$ of the form $\mathbf{E} \sim 1 / r$, which lends itself naturally to analysis via Cauchy's theorem. On the other hand, the

*mafurman@lbl.gov; http://mafurman.lbl.gov potential has a dependence on distance of the form $\Phi \sim$ $\ln r$, which is much more complicated to deal with in this formalism. For this reason, our results are not directly applicable to Hamiltonian analysis, since this requires an expression for the potential. While the analytic solution of the problem addressed here is known [1-3], our formalism, we believe, has the advantage of simplicity, ease of generalization, direct applicability to particle tracking and, in our opinion, elegance.

In Sec. II we recapitulate the results for free space and define our notation, which differs slightly from Ref. [4]. In Sec. III we define the image electric field produced by the conducting cylinder. We first establish a few properties valid for any charge distribution (not necessarily elliptical), and then obtain the explicit expression for the field for elliptical distributions. Section IV presents a few concrete examples, Sec. V contains a brief discussion concerning a generalization of the previous results to extended distributions, and the Appendix presents a few auxiliary results.

\section{RECAP: FREE SPACE}

We consider a static charge distribution that depends only on the two coordinates $x$ and $y$ and is infinitely long along the direction perpendicular to the $x-y$ plane. We write the volumetric charge density in the somewhat unconventional form $\lambda \rho(x, y)$, where $\lambda$ is the line charge density (with dimensions of charge/length) of the distribution along the direction perpendicular to the $x-y$ plane, and $\rho(x, y)$, with dimensions of $1 /$ area, is a real function normalized to unity,

$$
\int d x d y \rho(x, y)=1
$$

For this distribution, the electric field has only nonzero components in the $x-y$ plane,

$$
\mathbf{E}=\left(E_{x}, E_{y}, 0\right)
$$

The solution of Poisson's equation in free space, $\nabla$. $\mathbf{E}(\mathbf{x})=\lambda \rho(\mathbf{x}) / \epsilon_{0}$, at the observation point $\mathbf{x}=(x, y)$, subject to the condition $|\mathbf{E}(\mathbf{x})| \rightarrow 0$ as $|\mathbf{x}| \rightarrow \infty$, is 


$$
\mathbf{E}(\mathbf{x})=\frac{\lambda}{2 \pi \epsilon_{0}} \int d^{2} \mathbf{x}^{\prime} \frac{\mathbf{x}^{\prime}-\mathbf{x}}{\left|\mathbf{x}-\mathbf{x}^{\prime}\right|^{2}} \rho\left(x^{\prime}, y^{\prime}\right),
$$

where $\epsilon_{0}$ is the vacuum permittivity. We now define the "2D electric field" $\left(\mathcal{E}_{x}, \mathcal{E}_{y}\right)$, with dimensions of $1 /$ length, via

$$
\mathbf{E} \equiv \frac{\lambda}{4 \pi \epsilon_{0}}\left(\mathcal{E}_{x}, \mathcal{E}_{y}, 0\right)
$$

and the " $2 \mathrm{D}$ complex electric field" $\mathcal{E}$ via

$$
\mathcal{E} \equiv \mathcal{E}_{x}+i \mathcal{E}_{y}
$$

Equation (3) is then straightforwardly rewritten in the form

$$
\mathcal{E}(z)=2 \int d^{2} z^{\prime} \frac{\rho\left(z^{\prime}\right)}{\bar{z}-\bar{z}^{\prime}},
$$

where $z \equiv x+i y, z^{\prime} \equiv x^{\prime}+i y^{\prime}$, the bar denotes complex conjugation, and $d^{2} z^{\prime} \equiv d x^{\prime} d y^{\prime}$. We use the notation $\rho\left(z^{\prime}\right)$ rather than the more explicit form $\rho\left(x^{\prime}, y^{\prime}\right)$ for compactness of notation; it should always be kept in mind, however, that $\rho\left(z^{\prime}\right)$ is a real function of $x^{\prime}$ and $y^{\prime}$.

Clearly, $\mathbf{E}(\mathbf{x})$ and $\mathcal{E}(z)$ contain exactly the same information. The essential advantage of Eq. (6) over Eq. (3), however, is that Eq. (6) allows the power of complex calculus, particularly Cauchy's theorem, to be brought to bear on the computation of the field [4].

We consider first elliptical charge distributions centered at $x=y=0$, namely, those for which $\rho(x, y)$ depends on $x$ and $y$ only through the single dimensionless variable

$$
t=\frac{x^{2}}{a^{2}}+\frac{y^{2}}{b^{2}}
$$

rather than on $x$ and $y$ separately. We consider in this article only distributions of finite extent, so that $a$ and $b$ represent the semiaxes of the ellipse representing the edge of the distribution. This implies that $t$ is in the range $0 \leq t \leq 1$ or, equivalently, $\rho(t)=0$ for $t>1$ (we assume, without any loss of generality that $a \geq b$ ). This class of distributions can be expressed in the general form

$$
\rho(z)=\int_{0}^{\infty} d t \rho(t) \delta\left(\frac{x^{2}}{a^{2}}+\frac{y^{2}}{b^{2}}-t\right)
$$

so that Eq. (1) yields

$$
\int d^{2} z \rho(z)=\pi a b \int_{0}^{\infty} d t \rho(t)=1 .
$$

Defining the dimensionless density $\hat{\rho}(t) \equiv \pi a b \rho(t)$, which is normalized to unity,

$$
\int_{0}^{\infty} d t \hat{\rho}(t)=1
$$

then the basic result of Ref. [4] is ${ }^{1}$

\footnotetext{
${ }_{\mathcal{E}}{ }^{1}$ In Ref. [4] we included the line density $\lambda$ in the definition of
}

$$
\mathcal{E}(z)=\int_{0}^{|\xi|^{2}} d t \frac{2 \hat{\rho}(t)}{\sqrt{\bar{z}^{2}-t g^{2}}}
$$

where $g^{2} \equiv a^{2}-b^{2}$ and $\xi \equiv x / a+i y / b$.

Now if the elliptical 2D density is centered at the point $\left(x_{0}, y_{0}\right)$, then

$$
\rho(z)=\int_{0}^{\infty} d t \rho(t) \delta\left(\frac{\left(x-x_{0}\right)^{2}}{a^{2}}+\frac{\left(y-y_{0}\right)^{2}}{b^{2}}-t\right),
$$

therefore Eqs. (6) and (11) yield, upon a shift of integration variable $z^{\prime} \rightarrow z^{\prime}+z_{0}$,

$$
\mathcal{E}_{d}(z)=\int_{0}^{T} d t \frac{2 \hat{\rho}(t)}{\sqrt{\left(\bar{z}-\bar{z}_{0}\right)^{2}-\overline{t g}^{2}}},
$$

where $\quad z_{0} \equiv x_{0}+i y_{0}, \quad T \equiv\left|\xi-\xi_{0}\right|^{2}, \quad$ and $\quad \xi_{0} \equiv$ $x_{0} / a+i y_{0} / b$. We have appended the subscript " $d$ " to $\mathcal{E}$ to emphasize that this is the "direct" field, as opposed to the image field produced by the cylindrical boundary, which we address below.

The integral in Eq. (13) can be explicitly carried out for a significant class of interesting densities $\hat{\rho}(t)$; several examples for $z_{0}=0$ are presented in Ref. [4]. For $z_{0} \neq 0$, the expressions for $\mathcal{E}_{d}(z)$ are obtained from those for $z_{0}=0$ by the simple replacement $(x, y) \rightarrow\left(x-x_{0}, y-y_{0}\right)$.

The complex square root in Eqs. (11) and (13) is made well defined by a specific choice of the Riemann cut topology in the complex- $z$ plane. Only one of the two possible topologies, namely, the one in which the two Riemann cuts emanating out of the foci of the ellipse are joined together, yields the physically correct results [4]; see also Appendix A 1.

\section{CIRCULAR CYLINDER}

\section{A. Generic distributions}

A point charge at location $z$ inside a cylinder of radius $R$ centered at the origin of the $x-y$ plane has an image point charge of the same magnitude and opposite sign located at point $z_{i}$ outside the cylinder given by

$$
z_{i}=\frac{R^{2}}{\bar{z}}
$$

Therefore, the complex electric field at an observation point $z$ inside the cylinder produced by a unit point charge at location $z^{\prime}$, also inside the cylinder, is the Green's function [5]

$$
G\left(z, z^{\prime}\right)=\frac{2}{\bar{z}-\bar{z}^{\prime}}-\frac{2}{\bar{z}-\bar{z}_{i}^{\prime}},
$$

where $\bar{z}_{i}^{\prime} \equiv R^{2} / z^{\prime}$, hence the field for a general distribution $\rho(z)$ contained inside the cylinder is given by

$$
\mathcal{E}(z)=\int d^{2} z^{\prime} \mathcal{G}\left(z, z^{\prime}\right) \rho\left(z^{\prime}\right)
$$


The perfect-conductor boundary condition, namely that the vector $\left(\mathcal{E}_{x}, \mathcal{E}_{y}\right)$ must be perpendicular to the surface at the cylindrical boundary, $\left(\mathcal{E}_{x}, \mathcal{E}_{y}\right) \times(x, y)=0$, can be succinctly expressed in the form

$$
\operatorname{Im}[\bar{z} \mathcal{E}(z)]=0 .
$$

It is sufficient to verify this property for $G\left(z, z^{\prime}\right)$ for any fixed $z^{\prime}$ inside the cylinder and for any $z$ on the boundary, as Eq. (16) is a simple superposition of such contributions with the real weight $\rho\left(z^{\prime}\right)$. Substituting Eq. (15) into the left-hand side of Eq. (17) readily yields $\operatorname{Im}\left[\bar{z} G\left(z, z^{\prime}\right)\right]=0$ upon setting $z=R e^{i \theta}$ for arbitrary real $\theta$.

Note that, although the integral in Eq. (16) is over the entire complex- $z^{\prime}$ plane, it is effectively confined to the region where $\rho\left(z^{\prime}\right)$ is nonvanishing. An alternative expression for the field can be given by finding the image distribution $\rho_{i}(z)$ and adding up the contributions to $\mathcal{E}(z)$ from both $\rho(z)$ and $\rho_{i}(z)$ as if they were two independent, physical charge distributions,

$$
\mathcal{E}(z)=\int d^{2} z^{\prime} \frac{2}{\bar{z}-\bar{z}^{\prime}}\left[\rho\left(z^{\prime}\right)+\rho_{i}\left(z^{\prime}\right)\right] .
$$

Since the integral in Eq. (18) is over the entire complex- $z^{\prime}$

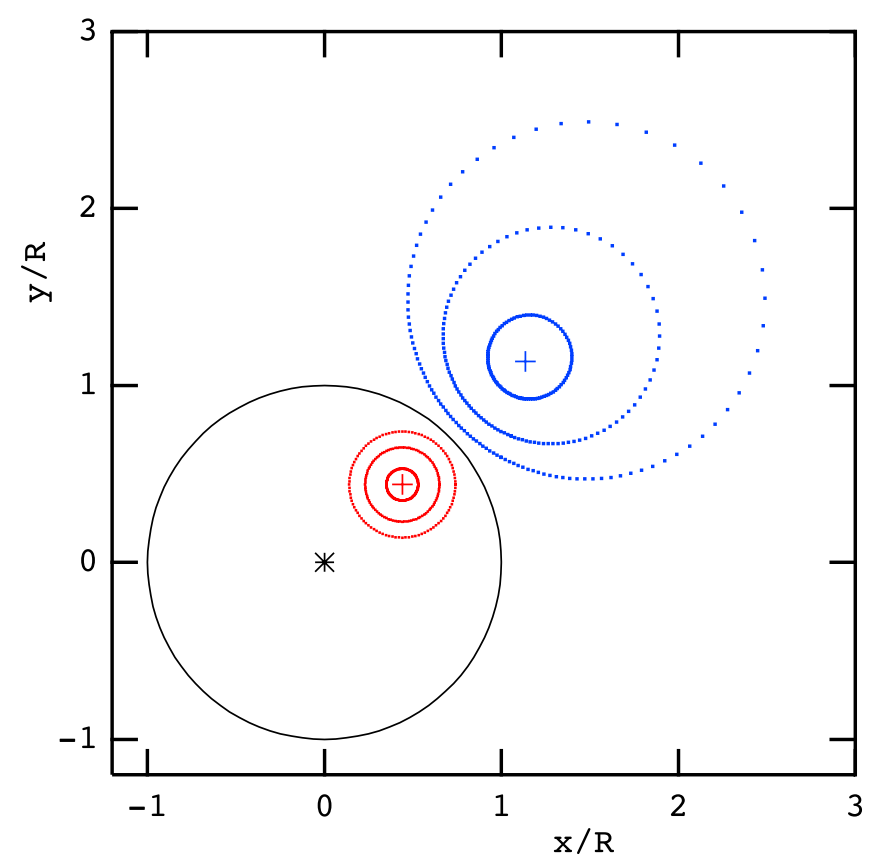

FIG. 1. (Color) The black circle represents the cross section of a perfectly conducting circular cylinder of radius $R$ centered at the origin. The image of any given circle (red) is a circle (blue), but the image of a 2D round charge distribution, represented by the red concentric level circles, is not a round charge distribution because the images of the various level circles are not concentric: the charge density of the image distribution is higher at the edge closer to the cylinder than at the farther edge, following the inverse 4th power of the distance, according to Eq. (19). The image of the center of any given circle is not the center of the image of the circle; see Eq. (A5). plane, the first term picks up only the contribution from the direct charge density $\rho\left(z^{\prime}\right)$, which is inside the cylinder, while the second term picks up only the contribution from the image charge density $\rho_{i}\left(z^{\prime}\right)$, which is outside the cylinder. A straightforward change of integration variable in Eqs. (15) and (16) yields

$$
\rho_{i}\left(z_{i}\right)=-\frac{R^{4}}{\left|z_{i}\right|^{4}} \rho(z),
$$

where $z_{i}$ and $z$ are related by Eq. (14). Sample images of round and elliptical distributions are shown in Figs. 1 and 2; see also Appendix A 2.

The complex image field $\mathcal{E}_{i}(z)$, namely, the second term in Eqs. (15) and (16),

$$
\mathcal{E}_{i}(z)=-2 \int d^{2} z^{\prime} \frac{\rho\left(z^{\prime}\right)}{\bar{z}-\bar{z}_{i}^{\prime}},
$$

is an analytic function of $\bar{z}$ when $z$ is inside the cylinder, as it should be according to the discussion in Sec. 1 of Ref. [4] (see also Appendix A 3). Indeed, substituting $\bar{z}_{i}^{\prime}=R^{2} / z^{\prime}$ in Eq. (20) yields the Taylor expansion about the origin

$$
\mathcal{E}_{i}(z)=\frac{2}{R} \sum_{n=0}^{\infty} M_{n+1}\left(\frac{\bar{z}}{R}\right)^{n},
$$

where $M_{n}$ is the $n$th moment of $\rho(z)$ normalized to $R^{n}$,

$$
M_{n} \equiv \frac{\left\langle z^{n}\right\rangle}{R^{n}}=\frac{1}{R^{n}} \int d^{2} z \rho(z) z^{n}
$$

In particular, $\mathcal{E}_{i}(z)$ has the curiously simple property

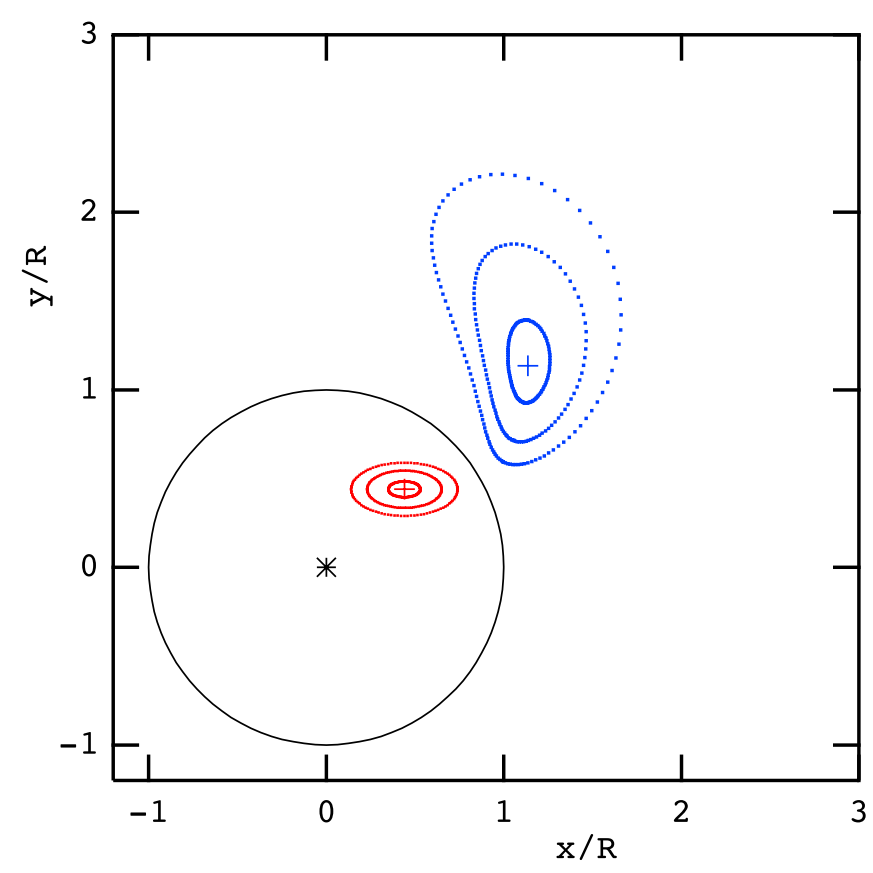

FIG. 2. (Color) The image of an elliptical distribution (red) is a mussel-shell shape distribution (blue). The level curves of the image are quartic, not quadratic. 


$$
\mathcal{E}_{i}(0)=\frac{2\langle z\rangle}{R^{2}}
$$

valid for any $\rho(z)$.

The series (21) converges everywhere inside the cylinder, a fact that follows from standard convergence tests upon noticing that $\left|M_{n}\right| \leq 1$. In general, however, the radius of convergence is larger than $R$ because $\mathcal{E}_{i}(z)$ is analytic everywhere outside the image density $\rho_{i}(z)$. The radius of convergence of the series (21), therefore, is the distance of closest approach to the origin of the edge of $\rho_{i}(z)$.

\section{B. Elliptical distributions}

We can now compute the complex image field $\mathcal{E}_{i}(z)$ at an observation point $z$ inside the cylinder of radius $R$ produced by the elliptical distribution (12) that is also wholly contained within the cylinder. Inserting $z_{i}^{\prime}=R^{2} / \bar{z}^{\prime}$ and $z_{i}=R^{2} / \bar{z}$ yields

$$
\frac{1}{\bar{z}-\bar{z}_{i}^{\prime}}=\frac{1}{\bar{z}}-\frac{z_{i} / \bar{z}}{z_{i}-z^{\prime}}
$$

therefore

$$
\begin{aligned}
\mathcal{E}_{i}(z) & =-\frac{2}{\bar{z}}+\frac{2 z_{i}}{\bar{z}} \int d^{2} z^{\prime} \frac{\rho\left(z^{\prime}\right)}{z_{i}-z^{\prime}} \\
& =-\frac{2}{\bar{z}}+\frac{2 z_{i}}{\bar{z}} \int_{0}^{T_{i}} d t \frac{\hat{\rho}(t)}{\sqrt{\left(z_{i}-z_{0}\right)^{2}-t g^{2}}}
\end{aligned}
$$

where the last equality follows from the same steps described in Ref. [4], and $T_{i}$ is given by

$$
T_{i}=\left(\frac{x_{i}-x_{0}}{a}\right)^{2}+\left(\frac{y_{i}-y_{0}}{b}\right)^{2} .
$$

Since $z_{i}$ is always outside the charge distribution (in fact outside the cylinder), then $T_{i}>1$ for any $z$, hence $T_{i}$ can be effectively replaced by 1 in the integral over $t$. Substituting $z_{i}=R^{2} / \bar{z}$ yields

$$
\mathcal{E}_{i}(z)=-\frac{2 z_{i}}{R^{2}}+\frac{2 z_{i}^{2}}{R^{2}} \int_{0}^{1} d t \frac{\hat{\rho}(t)}{\sqrt{\left(z_{i}-z_{0}\right)^{2}-\overline{t g}^{2}}},
$$

which constitutes the central result of this article.

Despite its singular appearance, Eq. (27) is regular in the limit $z \rightarrow 0$ (corresponding to $z_{i} \rightarrow \infty$ ), yielding

$$
\mathcal{E}_{i}(0)=\frac{2 z_{0}}{R^{2}}=\frac{2}{\bar{z}_{0 i}}
$$

for any $\hat{\rho}(t)$, in agreement with the general result (23). The expression for $\left\langle z^{n}\right\rangle$ is derived in Appendix A 4.

The final general result for the 2D complex electric field is therefore $\mathcal{E}(z)=\mathcal{E}_{d}(z)+\mathcal{E}_{i}(z)$, where the direct and image fields are given by Eqs. (13) and (27), respectively.

\section{EXAMPLES}

\section{A. Point charge}

As a simple first case, consider a point charge at $z_{0}$, corresponding to $\hat{\rho}(t)=\delta(t)$. Using the appropriate Riemann cut topology yields

$$
\mathcal{E}(z)=\frac{2}{\bar{z}-\bar{z}_{0}}-\frac{2}{\bar{z}}+\frac{2 z_{i} / \bar{z}}{z_{i}-z_{0}}=\frac{2}{\bar{z}-\bar{z}_{0}}-\frac{2}{\bar{z}-\bar{z}_{0 i}},
$$

where $z_{0 i}=R^{2} / \bar{z}_{0}$ is the image of $z_{0}$, in agreement with Eq. (15).

\section{B. Round distribution}

Setting $g=0$ in Eqs. (13) and (27) yields

$$
\mathcal{E}(z)=\frac{2 Q(z)}{\bar{z}-\bar{z}_{0}}-\frac{2}{\bar{z}-\bar{z}_{0 i}},
$$

where

$$
Q(z) \equiv \int_{0}^{T} d t \hat{\rho}(t)
$$

and where $T=\left|z-z_{0}\right|^{2} / a^{2}$. If $z$ is outside $\rho(z)$, then $T>$ 1 hence $Q(z)=1$, and Eq. (30) reduces to Eq. (29). This is the well-known result that, outside a round distribution, the field behaves as if the distribution were pointlike with all its charge concentrated at its center.

\section{Uniformly charged ellipse}

For a uniformly charged elliptical distribution, given by $\hat{\rho}(t)=\theta(1-t)$, we obtain from Eq. (27)

$$
\begin{aligned}
\mathcal{E}_{i}(z) & =-\frac{2}{\bar{z}}+\frac{4 z_{i} / \bar{z}}{z_{i}-z_{0}+\sqrt{\left(z_{i}-z_{0}\right)^{2}-g^{2}}} \\
& =\frac{2 z_{i}}{R^{2}}\left[\frac{z_{i}+z_{0}-\sqrt{\left(z_{i}-z_{0}\right)^{2}-g^{2}}}{z_{i}-z_{0}+\sqrt{\left(z_{i}-z_{0}\right)^{2}-g^{2}}}\right],
\end{aligned}
$$

while the direct field is obtained from Eq. (13) [4],

$$
\mathcal{E}_{d}(z)= \begin{cases}\frac{4\left(\xi-\xi_{0}\right)}{a+b}, & \left|\xi-\xi_{0}\right| \leq 1 \\ \frac{4}{\bar{z}-\bar{z}_{0}+\sqrt{\left(\bar{z}-\bar{z}_{0}\right)^{2}-g^{2}}}, & \left|\xi-\xi_{0}\right| \geq 1,\end{cases}
$$

where $\xi-\xi_{0}=\left(x-x_{0}\right) / a+i\left(y-y_{0}\right) / b$.

For more complicated distributions, for example those for which $\hat{\rho}(t)$ is a polynomial in $t$, the integral over $t$ in Eqs. (13) and (27) can be done straightforwardly by recursion. The results can generally be expressed quite compactly in terms of $\bar{z}-\bar{z}_{0}$ and the auxiliary complex variables $\xi-\xi_{0}$ and $\omega-\omega_{0} \equiv b\left(x-x_{0}\right) / a+i a(y-$ $\left.y_{0}\right) / b$, as exemplified by the parabolic distribution $\hat{\rho}(t)=$ $2(1-t)$ in Ref. [4].

\section{DISCUSSION}

For charge distributions that are mathematically infinite in extent but in practice well localized within the cylinder, 
Eq. (27) yields a good approximation for $\mathcal{E}_{i}(z)$ by replacing the top limit of the integral over $t$ by $\infty$. For example, for a bi-Gaussian distribution of rms sizes $\sigma_{x}, \sigma_{y} \ll R$ whose center is at a distance $d$ of closest approach to the cylinder boundary such that $d \gg \sigma_{x}, \sigma_{y}, \mathcal{E}_{i}(z)$ can be expressed in terms of the complex error function, similarly to the direct field $\mathcal{E}_{d}(z)$, by choosing $\hat{\rho}(t)=\frac{1}{2} e^{-t / 2}[4,6]$.

\section{ACKNOWLEDGMENTS}

I am indebted to J.D. Jackson for valuable comments and to J. Zhou for bringing Refs. [1-3] to my attention. This work was supported by U.S. DOE under Contract No. DE-AC02-05CH11231 and by the US-LHC Accelerator Research Program (US-LARP).

\section{APPENDIX: MISCELLANEOUS ITEMS}

\section{Computational issues}

In the above expressions for the complex electric field we find square roots of the form

$$
S(w) \equiv \sqrt{w^{2}-g^{2}},
$$

where $w$ is a generic complex variable representing any of the variables $\bar{z}, \bar{z}-\bar{z}_{0}, z_{i}-z_{0}$, etc. We have found that, when numerically evaluating square roots of this type, FORTRAN and other programs that feature complex arithmetic yield the incorrect result because they select, by default, the incorrect Riemann cut. One way to get around this problem, which we have found to be effective, is to rewrite $S(w)$ in the form

$$
S(w)=w \sqrt{1-g^{2} / w^{2}} .
$$

In this case $S(w)$ is, usually, properly evaluated. In particular, this expression yields $S(w)=w$ when $g \rightarrow 0$, which corresponds precisely to the function defined by the proper Riemann cut topology in this limit [4]. In general, it is desirable in practice to verify that $S(w)$ satisfies the required odd-parity property $S(-w)=-S(w)$ and the mirror symmetry properties $\operatorname{Re} S\left(w_{x},-w_{y}\right)=+\operatorname{Re} S\left(w_{x}, w_{y}\right)$ and $\operatorname{Im} S\left(w_{x},-w_{y}\right)=-\operatorname{Im} S\left(w_{x}, w_{y}\right)$ and their permutations, for $w$ in any quadrant (here $w_{x}$ and $w_{y}$ are the real and imaginary parts of $w$, respectively). Expression (A2), of course, requires an exception handling at $w=0$, a very minor complication.

\section{Linear fractional mappings}

Aside from the inconsequential complex conjugation, expression (14) is a particular example of a "linear fractional mapping" in the complex plane [7], whose general form is ${ }^{2}$

\footnotetext{
${ }^{2}$ We stick to the traditional but unfortunate convention of using the adjective "linear" in the name.
}

$$
z_{i}=\frac{\alpha z+\beta}{\gamma z+\delta}
$$

where $\alpha, \beta, \gamma$, and $\delta$ are, in general, complex constants satisfying $\gamma \neq 0$ and $\alpha \delta-\beta \gamma \neq 0$. An interesting property of Eq. (A3) is that it maps generic circles and straight lines into circles (but does not map ellipses into ellipses). ${ }^{3}$ In particular, the image of a circle of radius $r$ centered at $z_{0}$, $\left|z-z_{0}\right|=r$, produced by the simple image mapping (14) is the circle $\left|z_{i}-c_{i}\right|=r_{i}$, where the center $c_{i}$ and radius $r_{i}$ are given by

$$
c_{i}=\frac{R^{2} z_{0}}{\left|z_{0}\right|^{2}-r^{2}}, \quad r_{i}=\frac{R^{2} r}{\left.|| z_{0}\right|^{2}-r^{2} \mid} .
$$

We note that the image of the center of the circle, $z_{0 i}$, is not the center of the image of the circle, $c_{i}$. Rather, they are related by

$$
\frac{1}{c_{i}}=\frac{1}{z_{0 i}}-\frac{(r / R)^{2}}{z_{0}}
$$

\section{Analyticity of $\mathcal{E}(z)$}

Consider a function $F(z)$, not necessarily analytic, that vanishes outside a closed domain $\mathcal{D}$ in the complex- $z$ plane. Then the function $f(z)$ defined by the integral

$$
f(z)=\int d^{2} z^{\prime} \frac{F\left(z^{\prime}\right)}{z-z^{\prime}},
$$

which we assume to exist, is analytic outside $\mathcal{D}$. To prove this elementary theorem, one has only to verify that the Cauchy-Riemann conditions, namely

$$
\frac{\partial f_{x}}{\partial x}=\frac{\partial f_{y}}{\partial y} \quad \text { and } \quad \frac{\partial f_{x}}{\partial y}=-\frac{\partial f_{y}}{\partial x},
$$

are valid for $f(z)$. Now since the integral in Eq. (A6) exists, one may bring $\partial / \partial x$ and $\partial / \partial y$ inside the integral, and the theorem reduces to proving that $\left(z-z^{\prime}\right)^{-1}$ is an analytic function of $z$. But this is indeed the case because (a) $(z-$ $\left.z^{\prime}\right)^{-1}$ satisfies the Cauchy-Riemann conditions for $z \neq z^{\prime}$, and (b) $z-z^{\prime}$ never vanishes because $z$ is outside $\mathcal{D}$.

By taking the complex conjugate of Eq. (A6), one concludes that $\bar{f}$ is an analytic function of $\bar{z}$ whenever $z$ (not $\bar{z}$ ) is outside $\mathcal{D}$. Therefore, using the expression

$$
\mathcal{E}_{i}(z)=2 \int d^{2} z^{\prime} \frac{\rho_{i}\left(z^{\prime}\right)}{\bar{z}-\bar{z}^{\prime}}
$$

one concludes that $\mathcal{E}_{i}(z)$ is an analytic function of $\bar{z}$ whenever $z$ is outside $\rho_{i}(z)$, while Eq. (6) implies that $\mathcal{E}_{d}(z)$ is an analytic function of $\bar{z}$ whenever $z$ is outside $\rho(z)$. The first statement is exemplified by Eq. (32) upon recalling that

\footnotetext{
${ }^{3}$ In exceptional cases, it maps circles into straight lines and straight lines into straight lines.
} 
$z_{i}=R^{2} / \bar{z}$, while the second is manifestly exemplified by Eq. (33) for the case $\left|\xi-\xi_{0}\right| \geq 1$.

\section{Moments of an elliptical distribution}

Inserting Eq. (12) into Eq. (22), we obtain

$$
\left\langle z^{n}\right\rangle=\int_{0}^{1} d t \hat{\rho}(t) P_{n}(t)
$$

where we have defined

$$
P_{n}(t) \equiv \frac{1}{\pi a b} \int d^{2} z z^{n} \delta\left(\frac{\left(x-x_{0}\right)^{2}}{a^{2}}+\frac{\left(y-y_{0}\right)^{2}}{b^{2}}-t\right)
$$

Making the shift of integration variable $z \rightarrow z+z_{0}$ followed by $(x, y)=r(a \cos \phi, b \sin \phi)$, we obtain

$$
\begin{aligned}
P_{n}(t)= & \int_{0}^{2 \pi} \frac{d \phi}{\pi} \int_{0}^{\infty} r d r\left(z_{0}+a r \cos \phi+i b r \sin \phi\right)^{n} \\
& \times \delta\left(r^{2}-t\right) \\
= & \int_{0}^{2 \pi} \frac{d \phi}{2 \pi}\left(z_{0}+a \sqrt{t} \cos \phi+i b \sqrt{t} \sin \phi\right)^{n} .
\end{aligned}
$$

Now changing the integration variable from $\phi$ to $\zeta \equiv e^{i \phi}$, we obtain a counterclockwise line integral over the unit circle in the complex- $\zeta$ plane,

$$
P_{n}(t)=\frac{1}{2 \pi i} \oint_{|\zeta|=1} \frac{d \zeta}{\zeta}\left(z_{0}+A \zeta+B \zeta^{-1}\right)^{n},
$$

where $\quad A \equiv(a+b) \sqrt{t} / 2 \quad$ and $\quad B \equiv(a-b) \sqrt{t} / 2$. Expanding the parentheses yields

$$
\begin{aligned}
\left(z_{0}+A \zeta+B \zeta^{-1}\right)^{n}= & \sum_{k=0}^{n} \sum_{m=0}^{k}\left(\begin{array}{l}
n \\
k
\end{array}\right)\left(\begin{array}{l}
k \\
m
\end{array}\right) z_{0}^{n-k}(A \zeta)^{m} \\
& \times\left(B \zeta^{-1}\right)^{k-m} .
\end{aligned}
$$

By Cauchy's theorem only the terms $\propto \zeta^{0}$ in Eq. (A13) yield a nonzero contribution to Eq. (A12). This implies $m=k-m$ hence $m=k / 2$, hence $k=$ even. Therefore, setting $k=2 \ell$, we obtain

$$
P_{n}(t)=n ! z_{0}^{n} \sum_{\ell=0}^{[n / 2]} \frac{t^{\ell}}{(n-2 \ell) ! \ell !^{2}}\left(\frac{g}{2 z_{0}}\right)^{2 \ell},
$$

where $[n / 2]$ is the largest integer $\leq n / 2$. Equation (A9) implies that the moment $\left\langle z^{n}\right\rangle$ is obtained from Eq. (A14) by the simple replacement $t^{\ell} \rightarrow\left\langle t^{\ell}\right\rangle$, where

$$
\left\langle t^{\ell}\right\rangle \equiv \int_{0}^{1} d t \hat{\rho}(t) t^{\ell}
$$

hence

$$
M_{n}=n !\left(\frac{z_{0}}{R}\right)^{n} \sum_{\ell=0}^{[n / 2]} \frac{\left\langle t^{\ell}\right\rangle}{(n-2 \ell) ! \ell !^{2}}\left(\frac{g}{2 z_{0}}\right)^{2 \ell}
$$

For a centered distribution $\left(z_{0}=0\right)$, Eq. (A16) yields

$$
M_{n}=\frac{n !}{(n / 2) !^{2}}\left(\frac{g}{2 R}\right)^{n}\left\langle t^{n / 2}\right\rangle
$$

if $n=$ even, and $M_{n}=0$ if $n=$ odd, while for a round distribution $(g=0)$ Eq. (A16) yields

$$
M_{n}=\left(\frac{z_{0}}{R}\right)^{n}
$$

[1] B. L. Qian, J. Zhou, and C. Chen, Phys. Rev. ST Accel. Beams 6, 014201 (2003).

[2] J. Zhou, B. L. Qian, and C. Chen, Phys. Plasmas 10, 4203 (2003).

[3] M. Hess and C. Chen, Phys. Plasmas 9, 1422 (2002).

[4] M. A. Furman, Am. J. Phys. 62, 1134 (1994). Minor revisions and extensions of this publication can be found in http://mafurman.lbl.gov/LBL-34682.pdf.

[5] See, for example, J.D. Jackson, Classical Electrodynamics (3rd ed.) (Wiley, New York, 1999), Chap. 2.

[6] M. Bassetti and G. A. Erskine, CERN-ISR-TH/80-06.

[7] See, for example, J.W. Dettman, Applied Complex Variables (Dover, New York, 1984), Chap. 2; original publication: Macmillan, New York, 1965. 